ARTICLE

https://doi.org/10.1038/s41467-019-09211-z

\title{
Two-dimensional molecular brush-functionalized porous bilayer composite separators toward ultrastable high-current density lithium metal anodes
}

\author{
Chuanfa Li ${ }^{1}$, Shaohong Liu (i) ${ }^{1}$, Chenguang Shi ${ }^{1}$, Ganghao Liang ${ }^{1}$, Zhitao Lu', Ruowen Fu ${ }^{1}$ \& Dingcai Wu ${ }^{1}$
}

Lithium metal batteries have been considerably limited by the problems of uncontrolled dendritic lithium formation and the highly reactive nature of lithium with electrolytes. Herein, we have developed functional porous bilayer composite separators by simply blade-coating polyacrylamide-grafted graphene oxide molecular brushes onto commercial polypropylene separators. Our functional porous bilayer composite separators integrate the lithiophilic feature of hairy polyacrylamide chains and fast electrolyte diffusion pathways with the excellent mechanical strength of graphene oxide nanosheets and thus enable molecular-level homogeneous and fast lithium ionic flux on the surfaces of electrodes. As a result, dendritefree uniform lithium deposition with a high Coulombic efficiency (98\%) and ultralong-term reversible lithium plating/stripping (over $2600 \mathrm{~h}$ ) at a high current density $\left(2 \mathrm{~mA} \mathrm{~cm}{ }^{-2}\right)$ are achieved for lithium metal anodes. Remarkably, lithium metal anodes with an unprecedented stability of more than $1900 \mathrm{~h}$ cycling at an ultrahigh current density of $20 \mathrm{~mA} \mathrm{~cm}^{-2}$ are demonstrated. 
$\mathrm{R}$ esearch on high-performance electrochemical energy storage has been pursued worldwide to fulfill the needs of emerging high-energy-demand applications, such as portable electronics, electric vehicles, autonomous aircraft, and grid storage $^{1-4}$. Among the myriad electrochemical energy-storage technologies, lithium-ion batteries (LIBs) have monopolized the major markets since their commercialization in 1991 because of their intrinsic high-energy density, light weight, and minimal memory effect ${ }^{5}$. Unfortunately, due to the limited theoretical specific capacities of the widely used commercial anode $\left(\mathrm{LiC}_{6}\right)$ and cathode materials $\left(\mathrm{LiCoO}_{2}\right)$, the highest energy density that traditional LIBs can deliver even when fully developed is far from the demands of these emerging applications ${ }^{6-9}$. With the highest theoretical specific capacity $\left(3860 \mathrm{mAh} \mathrm{g}^{-1}, 10\right.$ times that of commercial graphite anodes) and the lowest redox potential $(-3.04 \mathrm{~V}$ vs. the standard hydrogen electrode) among all anode materials, lithium (Li) metal has long been regarded as the "Holy Grail" anode of Li-based batteries and is a promising candidate for next-generation energy-storage systems with high-energy cathode materials, such as sulfur and oxygen ${ }^{10-12}$.

Nevertheless, the application of $\mathrm{Li}$ metal anodes has been considerably limited because of uncontrolled dendritic Li formation and the highly reactive nature of $\mathrm{Li}^{13-15}$. Specifically, due to the unavoidable microscopic roughness of electrode surfaces, the electrical fields near the electrode surfaces are nonuniformly distributed during cell operation, which results in an inhomogeneous distribution of $\mathrm{Li}$ ions. As a result, more $\mathrm{Li}$ will be preferentially deposited around the protuberant tips with stronger electrical fields ${ }^{11,16}$. Notably, this preferential deposition behavior undergoes self-amplification with gradually increasing surface roughness and tip electrical field intensity, leading to the gradual formation of Li dendrites (Fig. 1a). The resultant Li dendrites can bridge the interelectrode spaces and thus cause internal short circuits in the cell, which gives rise to obvious safety limitations on the cells ${ }^{17}$. On the other hand, highly reactive Li metal can irreversibly decompose the solvent and salts to instantly form a solid electrolyte interphase (SEI) protecting layer on the Li surface. Such an SEI protecting layer is too brittle to alleviate the significant volume change of $\mathrm{Li}$ metal during plating/stripping processes, exposing fresh Li metal underneath to the electrolyte ${ }^{18}$. As a result, Li dendrite growth is accelerated at the exposed sites, and Li metal and electrolyte are continuously consumed through the formation of SEI layers over repeated plating/stripping processes, giving rise to a low Coulombic efficiency and poor cycling stability. Furthermore, these issues are more severe under the high-current densities that are needed due to the increasing demand of high-power devices.

In this context, numerous efforts have been devoted to addressing these issues and unlocking the full potential of $\mathrm{Li}$ metal anodes. One method is to optimize the liquid electrolytes by adjusting the electrolyte compositions ${ }^{19}$ or adding various electrolyte additives ${ }^{20-23}$. These additives can react rapidly with Li metal to modify the chemical composition of SEI layers and thus improve their stability and uniformity. Nevertheless, in most cases, the in situ formed protecting layers are still not strong enough to withstand the mechanical deformation induced by dendrite growth, and stable cycling is not sustainable over a long period of time due to the consumption of such additives ${ }^{24,25}$. Another method is to ex situ coat the surfaces of Li metal anodes by various artificial protective layers, such as polymeric grafted skin ${ }^{26}$, ultrathin $\mathrm{Al}_{2} \mathrm{O}_{3}$ layer ${ }^{27}$, and $\mathrm{LiF} / \mathrm{PVDF}-\mathrm{HFP}$ composite film ${ }^{28}$, which offer high chemical stability and mechanical strength, thus overcoming the fragility issue. However, the artificial protective layers often result in increased polarization voltages because of their relatively high resistance ${ }^{28}$. Additionally, ex situ coating procedures are usually tedious and dangerous because of the highly reactive nature of $\mathrm{Li}$ metal, which could make large-scale industrial operations difficult.

As an important part of cells, separators play a critical role in determining cell's electrochemical performance. It is believed that separator modification is more convenient than Li metal anode modification to achieve stable Li plating/stripping ${ }^{29}$. Recently, it has been proposed that high-modulus separators, such as solidstate electrolyte membranes and robust interlayers, can function as strong mechanical barriers to block dendrite growth ${ }^{22,30-35}$. Nevertheless, these mechanical barriers introduce the additional problem of large interfacial resistance, and the fundamental problem of uneven $\mathrm{Li}$ electrodeposition remains unsolved. On the other hand, a few reports have demonstrated that the use of lithiophilic separators with abundant polar functional groups can homogenize Li-ion flux on the electrode surfaces and thus promote uniform Li deposition ${ }^{36-39}$. Unfortunately, the mechanical strength of lithiophilic separators is rather poor and cannot readily withstand the large pressure induced by the substantial volume change of $\mathrm{Li}$ metal anodes ${ }^{12}$. Additionally, the stable plating/stripping of $\mathrm{Li}$ anodes can only be achieved under limited current densities (mostly no more than $2 \mathrm{~mA} \mathrm{~cm}^{-2}$ ). Therefore, it is imperative to develop advanced separators that simultaneously possess favorable mechanical strength, high ionic conductivity, and valuable lithiophilic features to boost the performance of Li metal anodes under very high current densities.

Herein, a class of functional porous bilayer composite separators is employed for the first time to regulate homogeneous $\mathrm{Li}$ deposition and achieve superstable $\mathrm{Li}$ metal anodes under ultrahigh current densities. Our advanced composite separators are facilely prepared by blade-coating two-dimensional (2D) molecular brushes (i.e., polyacrylamide-grafted graphene oxide nanosheets, GO-g-PAM) onto one side of commercial polypropylene (PP) separators, which is highly convenient for the large-scale production of modified separators. In the composite separators, the robust GO backbones of the molecular brushes improve the mechanical strength, while the hairy PAM chains on the GO surfaces with a large quantity of polar groups including $\mathrm{C}=\mathrm{O}$ and $\mathrm{N}-\mathrm{H}$ bonds provide high-concentration functional sites for the efficient adhesion and homogeneous distribution of $\mathrm{Li}$ ions at the molecular level. Moreover, the interspaces between the stacked 2D molecular brushes provide fast pathways for the diffusion of electrolytes. As a result, molecular-level homogeneous and fast $\mathrm{Li}$ ionic flux on the surface of electrodes is achieved with the porous bilayer composite separators, giving rise to dendrite-free uniform $\mathrm{Li}$ deposition with high Coulombic efficiencies and ultralong-term reversible Li plating/stripping under very high current densities (Fig. 1b).

\section{Results}

Preparation and characterization of 2D molecular brushes. The synthetic strategy of GO- $g$-PAM molecular brushes is schematically presented in Fig. 2a. The GO nanosheets are first modified by a-bromoisobutyryl bromide to introduce Br-containing initiation sites onto the surface. Afterward, hairy PAM chains are successfully grafted from the modified surface of GO nanosheets via surface-initiated atom-transfer radical polymerization (SI-ATRP), a very successful strategy for the preparation of well-defined surface polymers. The Fourier transform infrared (FTIR) spectra in Fig. 2b reveal that the PAM chains are successfully grafted from the GO nanosheets, as demonstrated by the presence of two new characteristic peaks of methylene groups at 2922 and $2853 \mathrm{~cm}^{-1}$ and the increased peak intensity of $\mathrm{C}=\mathrm{O}$ bonds at $1720 \mathrm{~cm}^{-1}$ for GO- $g$-PAM. The two strong peaks at 1598 and $770 \mathrm{~cm}^{-1}$ for GO- $g$-PAM can be ascribed to the inplane and out-of-plane scissoring bending vibrations of primary 
a
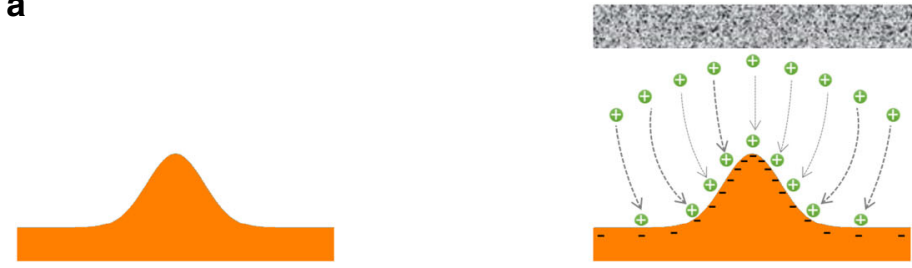

Charging
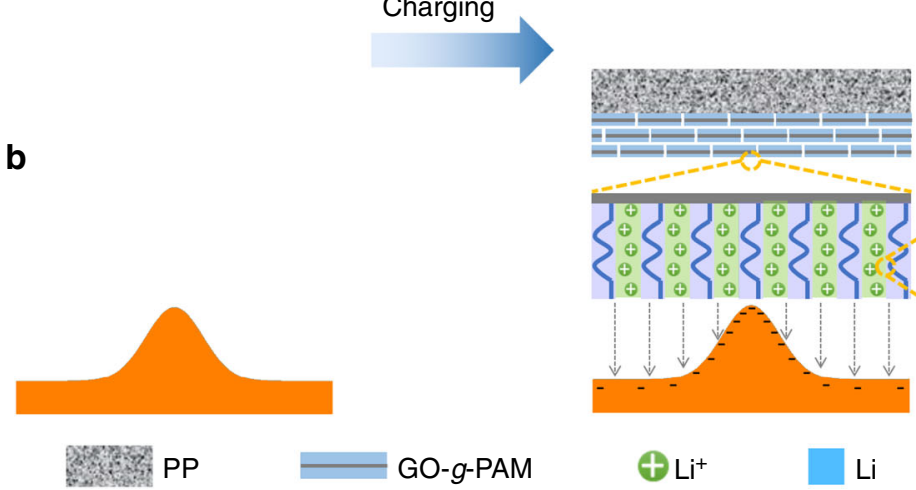

$\oplus \mathrm{Li}^{+}$

$\mathrm{Li}$

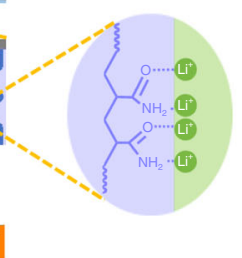

Current collector
Li plating
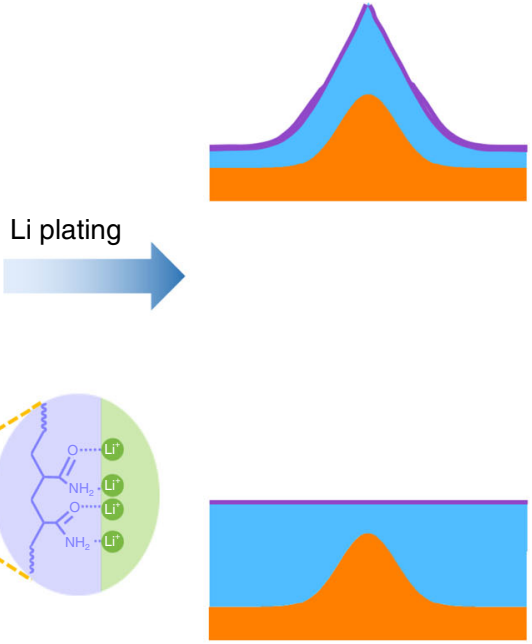

SEI

Fig. 1 Schematic diagram of Li deposition on electrodes with microscopic surface roughness. a Schematics showing Li deposition on an electrode with a PP separator. Because of the stronger electrical field, Li ions will aggregate near the protuberant tip, leading to the formation of Li dendrites. $\mathbf{b}$ Schematics showing Li deposition on an electrode with a GO-g-PAM@PP separator. The GO-g-PAM molecular brushes with a large quantity of polar functional groups $(\mathrm{C}=\mathrm{O}, \mathrm{N}-\mathrm{H})$ provide high-concentration functional sites for the efficient adhesion and homogeneous distribution of $\mathrm{Li}$ ions at the molecular level, giving rise to dendrite-free uniform Li deposition

a

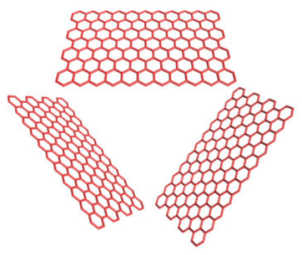

GO

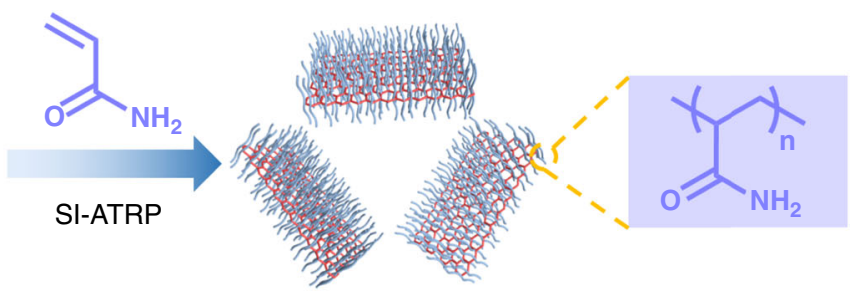

GO-g-PAM

b
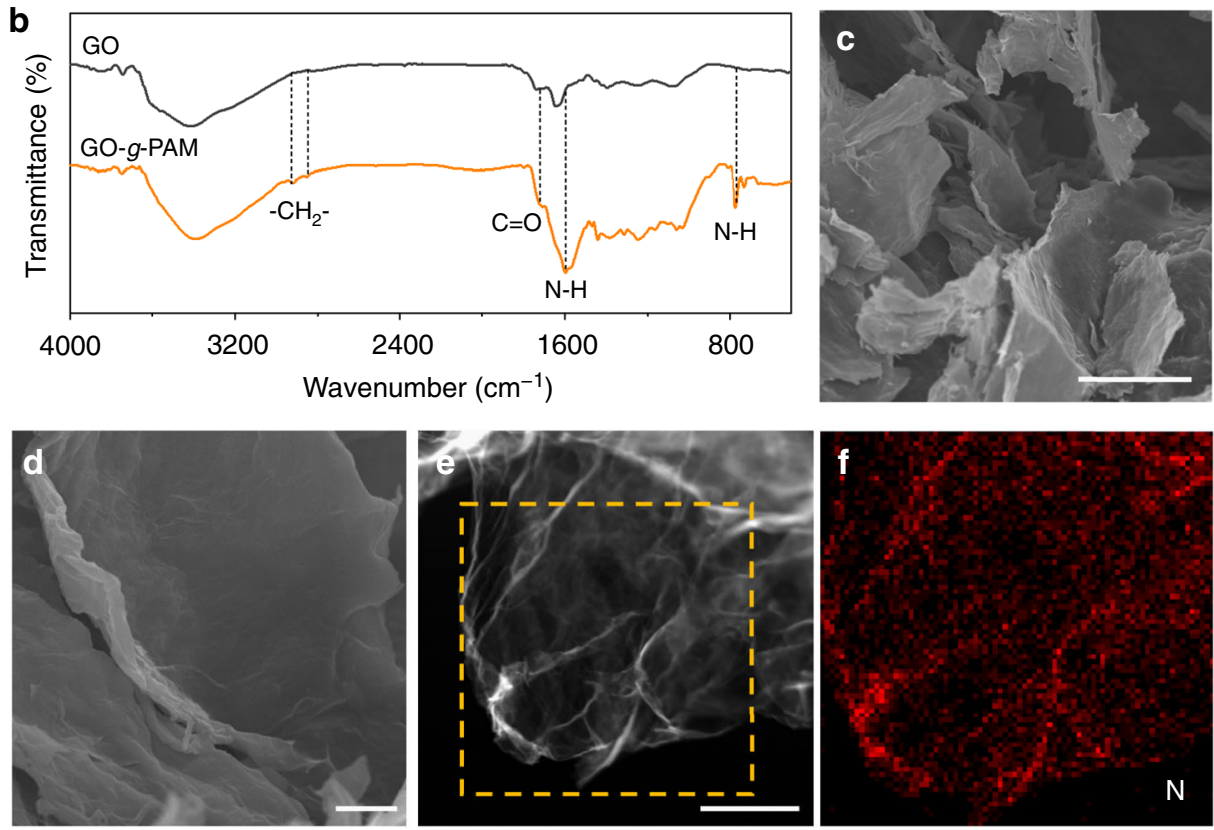

Fig. 2 Characterization of GO-g-PAM molecular brushes. a Schematic illustration of the synthesis of GO-g-PAM molecular brushes: modifying GO nanosheets by $\alpha$-bromoisobutyryl bromide to introduce Br-containing initiation sites and in situ grafting PAM chains from the modified surface of GO nanosheets via SI-ATRP. b FTIR spectra of GO and GO-g-PAM. c, d SEM images of GO-g-PAM. e, $\mathbf{f}$ Elemental mapping showing the molecular-level homogeneous distribution of $\mathrm{N}$ on GO nanosheets. Scale bars: (c) $20 \mu \mathrm{m}$, (d) $2 \mu \mathrm{m}$, and (e) $500 \mathrm{~nm}$ 
$\mathrm{NH}_{2}$ groups, respectively. The results demonstrate the presence of substantial polar functional groups, including $\mathrm{C}=\mathrm{O}$ and $\mathrm{NH}_{2}$ in GO- $g$-PAM. The nanomorphology of GO- $g$-PAM is investigated by scanning electron microscopy (SEM). As shown in Fig. 2c, d, the as-prepared GO-g-PAM molecular brushes present a typical 2D nanosheet morphology. No obvious polymer particles are observed on the surface, indicating homogeneous PAM grafting to the GO surface. Elemental mapping analysis of the GO- $g$-PAM molecular brushes also reveals the molecular-level homogeneous distribution of $\mathrm{N}$ (Fig. 2e, f), further confirming the uniform grafting of PAM chains and the homogeneous distribution of their acylamido groups. Therefore, benefiting from the unique $2 \mathrm{D}$ nanosheet structure of GO- $g$-PAM, the polar functional groups of the surface PAM chains can be largely exposed, which can facilitate homogeneous Li ionic flux and deposition.

Fabrication and characterization of functional porous bilayer composite separators. The GO- $g$-PAM molecular brushes are facilely coated onto one side of a commercial Celgard 2325 polypropylene (PP) separator with a blade to form a functional porous bilayer composite separator (GO- $g$-PAM@PP), whose functional porous coating layer results from the nanosheet interstacking of GO-g-PAM. As shown in Fig. 3a and Supplementary Fig. 1, the GO- $g$-PAM@PP separator can be bent or even rolled around a glass rod without any detachment. In contrast, the PAM-coated PP separator (PAM@PP) is very brittle, which leads to severe detachment and breaking of the coated PAM layer once it is slightly bent (Supplementary Fig. 2). Additionally, the atomic force microscope (AFM) Young's modulus mappings reveal that the GO-g-PAM@PP separator exhibits a higher Young's modulus than the PP and PAM@PP separators (Supplementary Fig. 3), suggestive of excellent mechanical strength enabled by the GO-g-PAM molecular brushes. A cross-section
SEM image of GO- $g$-PAM@PP shows that the coated GO- $g$-PAM layer adheres closely to the surface of the PP layer (Fig. 3b). The SEM images in Fig. 3c, d and Supplementary Fig. 4 show that both the GO-g-PAM-modified layer and the PP layer have welldeveloped pores. The pores in the functional layer can be ascribed to the interspaces between the interstacked nanosheets (Fig. 3c). Moreover, due to their large sheet size, GO-g-PAM nanosheets cannot enter the pores of the original PP separator during coating. Thus, the porous structure of the PP layer is retained very well in the GO-g-PAM@PP (Fig. 3d and Supplementary Fig. 4). $\mathrm{N}_{2}$ adsorption-desorption isotherm and density functional theory (DFT) pore size distribution (PSD) curve in Supplementary Fig. 5 further indicate that the GO-g-PAM@PP separator has numerous mesopores and macropores with a maximum PSD peak at $54 \mathrm{~nm}$. It should be noted that some large macropores may be too large and too open to cause capillary condensation and thus are not reflected in the PSD curve. Moreover, the large electrolyte uptake of the GO-g-PAM@PP separator also confirms its highly porous structure (Supplementary Fig. 6). These well-developed pores can provide fast pathways for $\mathrm{Li}$ ionic diffusion. As shown in Supplementary Fig. 7, the electrolyte can rapidly spread and permeate to the bottom side of the GO-g-PAM@PP separator when it is dropped onto the top GO-g-PAM coating.

The GO-g-PAM@PP separator demonstrates great affinity with the electrolyte, because of the substantial polar functional groups ${ }^{22}$. As shown in Supplementary Fig. 8, the contact angle of electrolyte on the GO-g-PAM@PP separator is almost zero, much lower than that on the PP separator $\left(23^{\circ}\right)$. This enhanced affinity with the electrolyte allows a good electrode-electrolyte contact and thus enhances the $\mathrm{Li}$ ionic transport near the electrode -electrolyte interface, leading to a decreased interface resistance between the Li electrode and electrolyte. More importantly, the strong lithiophilic characteristics of PAM chains also endow
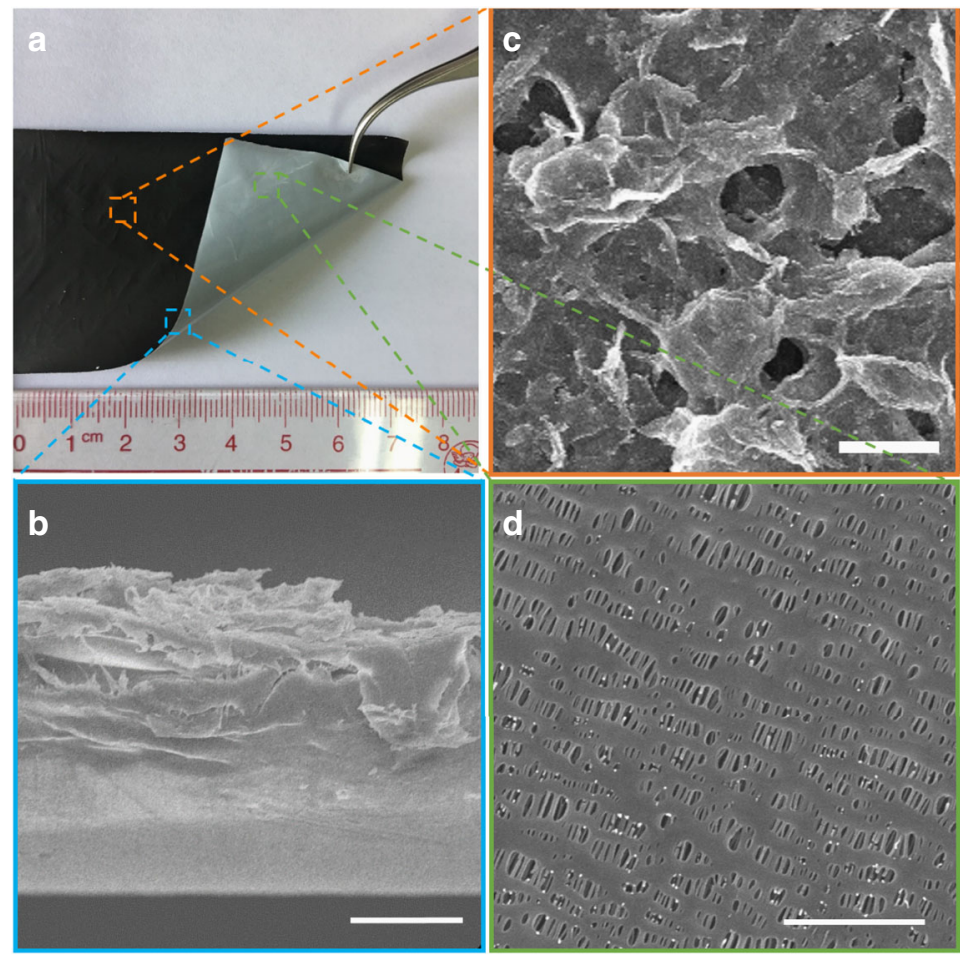

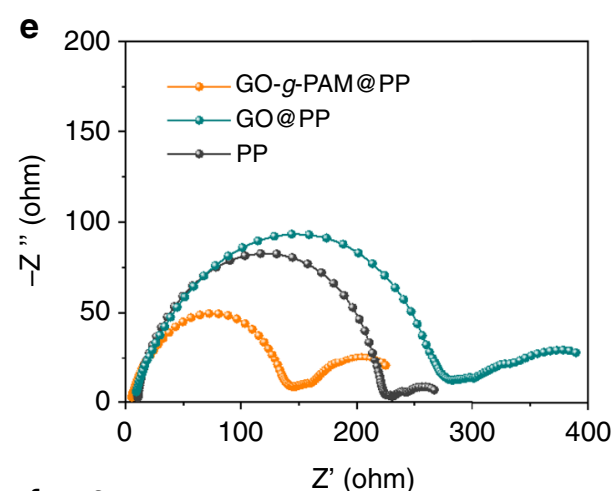

Fig. 3 Characterization of the GO-g-PAM@PP separator. a Digital photograph of a bent GO-g-PAM@PP separator. b Cross-section and (c) top-view SEM images of the GO-g-PAM@PP separator. d SEM image of the uncoated side of the GO-g-PAM@PP separator. e Nyquist plots of symmetric Li|Li cells with PP, GO@PP, and GO-g-PAM@PP separators. f LSV curves of asymmetric Li|stainless-steel cells with PP, GO@PP, and GO- $g$-PAM@PP separators at a scan rate of $5 \mathrm{mV} \mathrm{s}^{-1}$. Scale bars: (b) $20 \mu \mathrm{m}$, (c) $5 \mu \mathrm{m}$, and (d) $1 \mu \mathrm{m}$ 

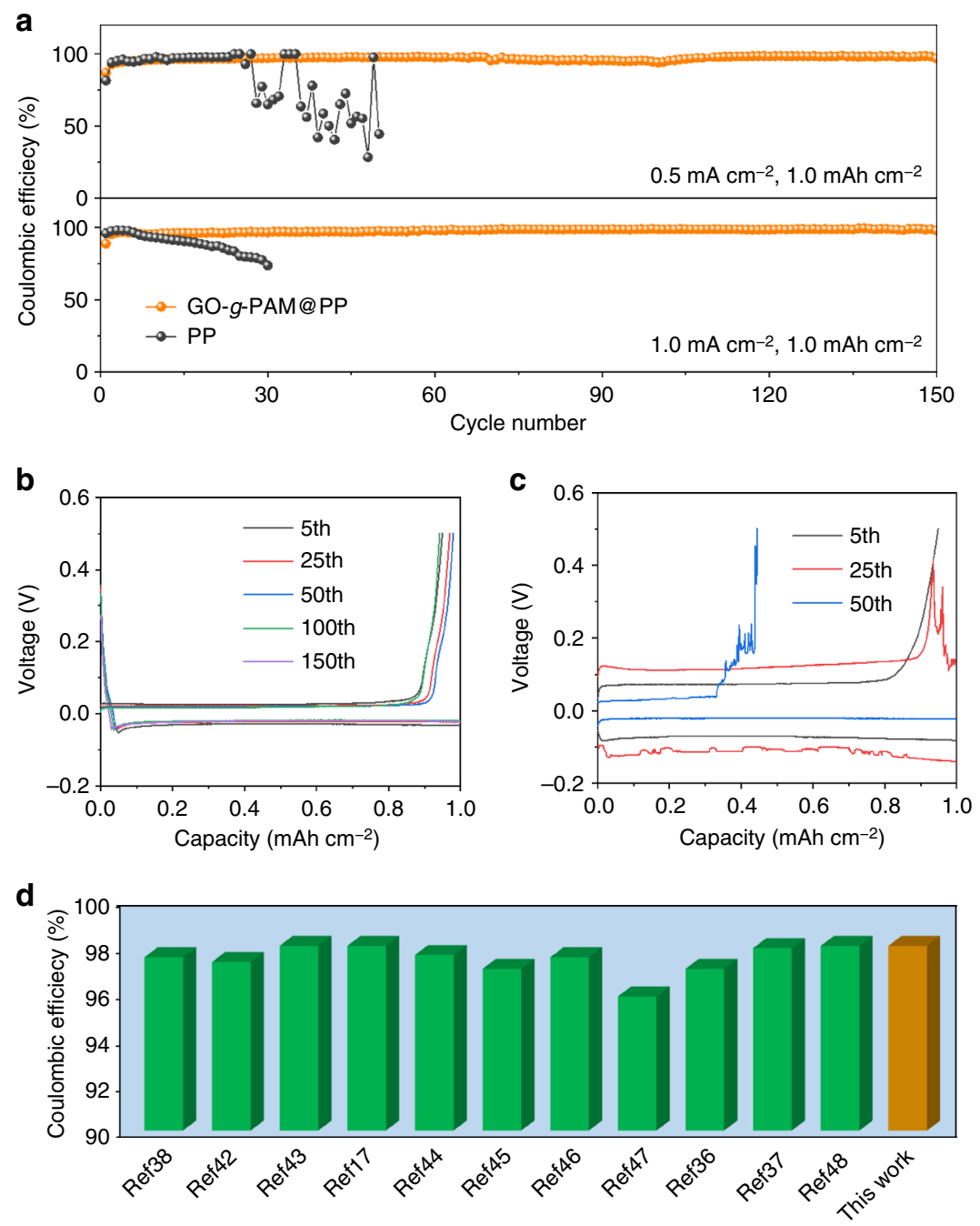

Fig. 4 Electrochemical performance of Li|Cu cells with GO-g-PAM@PP and PP separators. a Coulombic efficiencies of Li|Cu cells with GO-g-PAM@PP and PP separators with a cycling capacity of $1 \mathrm{mAh} \mathrm{cm}^{-2}$ at various current densities. Voltage profiles of Li plating/stripping processes in Li|Cu cells with (b) GO-g-PAM@PP and (c) PP separators with a cycling capacity of $1 \mathrm{mAh} \mathrm{cm}^{-2}$ at $0.5 \mathrm{~mA} \mathrm{~cm}^{-2}$. d Average Coulombic efficiency of a Li|Cu cell with GO-g-PAM@PP separator tested with a current density of $1 \mathrm{~mA} \mathrm{~cm}^{-2}$, compared with that of previously reported asymmetric cells with Li metal anodes stabilized by various strategies

the GO-g-PAM@PP separator with a significant electrokinetic pumping feature, which can accelerate the Li-ion transport and further decrease the battery resistance ${ }^{40}$. As shown in Fig. 3e, the symmetric Li|Li cell with the GO-g-PAM@PP separator demonstrates a considerably lower interfacial impedance than those with the PP separator and GO@PP separator, verifying the enhanced Li ionic transport kinetics. The cell with the GO@PP separator has a higher interfacial impedance than that with the PP separator, indicating the slow infiltration of the liquid electrolyte through the GO layers, which further demonstrates the important role of the surface PAM chains. A linear sweep voltammetry (LSV) test suggests that the GO-g-PAM@PP separator is stable up to $4.7 \mathrm{~V}$ vs. $\mathrm{Li}^{+} / \mathrm{Li}$, performance similar to that of the GO@PP separator yet slightly higher than that of the PP separator, indicating the high electrochemical stability of the GO-g-PAM coating (Fig. 3f). Moreover, the electrical conductivity tests reveal the electrically insulated feature of the GO- $g$-PAM@PP separator (Supplementary Fig. 9).

Electrochemical performance of GO- $g$-PAM@PP as a separator in $\mathrm{Li}$ metal batteries. Benefiting from the aforementioned unique physiochemical merits, the GO-g-PAM@PP separator is promising for regulating $\mathrm{Li}$ deposition and suppressing $\mathrm{Li}$ dendrite growth at a molecular scale. As shown in Supplementary Fig. 10a, b, typical mossy $\mathrm{Li}$ in the submicron size range with nonuniform structure is clearly observed after directly plating $1 \mathrm{mAh} \mathrm{cm}^{-2}$ of $\mathrm{Li}$ on the $\mathrm{Cu}$ foils in the cells with PP and GO@PP separators, respectively. In contrast, $\mathrm{Li}$ metal is homogeneously deposited on the $\mathrm{Cu}$ foil under identical conditions when GO-g-PAM@PP is used as the separator, leading to the formation of a dendrite-free morphology with a considerably flat surface (Supplementary Fig. 10c). The results demonstrate that the GO-g-PAM can facilitate the homogeneous redistribution of the $\mathrm{Li}$ ions that accumulate around the protuberant tips on the $\mathrm{Cu}$ foil (Supplementary Fig. 11), giving rise to uniform Li deposition.

The overall electrochemical performance of $\mathrm{Li} \mid \mathrm{Cu}$ cells with various separators is further investigated by a galvanostatic cycling test. The Li|Cu cells are first cycled from 0 to $1 \mathrm{~V}$ at $50 \mu \mathrm{A}$ for five cycles to stabilize the interfaces ${ }^{41}$. Notably, a pair of stable lithiation and delithiation plateaus corresponding to a small areal capacity of $\sim 0.1 \mathrm{mAh} \mathrm{cm}^{-2}$ are observed for the cell with the GO-g-PAM@PP separator but are absent for the cells with the PP and GO@PP separators (Supplementary Fig. 12), 
verifying the lithiophilic characteristics of the PAM molecular chains ${ }^{34}$. Thus, the Li nucleation overpotential, based on the difference between the tip voltage and the flat voltage of the first Li plating voltage profile, is only $33 \mathrm{mV}$ for the GO-g-PAM@PP separator, much lower than those of the PP and GO@PP separators (Supplementary Fig. 13).

The cycling Coulombic efficiencies of the $\mathrm{Li} \mid \mathrm{Cu}$ cells with various separators are further examined. Herein, Li metal with a total capacity of $1 \mathrm{mAh} \mathrm{cm}^{-2}$ is first plated on the $\mathrm{Cu}$ foil working electrodes, and then, the deposited $\mathrm{Li}$ is completely stripped from the $\mathrm{Cu}$ foils with a cutoff voltage of $0.5 \mathrm{~V}$ at the same current density. As shown in Fig. $4 \mathrm{a}, \mathrm{b}$, the $\mathrm{Li} \mid \mathrm{Cu}$ cell with the GO- $g$-PAM@PP separator demonstrates a steady Coulombic efficienciy of $97 \%$ with stable plating/stripping voltage profiles for more than 150 cycles at a current density of $0.5 \mathrm{~mA} \mathrm{~cm}^{-2}$. In comparison, the cells with the PP and GO@PP separators display significantly inferior electrochemical cycling properties with severely fluctuating plating/stripping voltage profiles and Coulombic efficiencies during the cycles (Fig. 4a, c and Supplementary Fig. 14); these fluctuations can be ascribed to the continuous breakdown/repair of SEI, which consumes both Li and electrolyte. In addition, the $\mathrm{Li} \mid \mathrm{Cu}$ cell with the GO-g-PAM@PP separator shows a smaller and more stable voltage hysteresis than those with the PP and GO@PP separators (Supplementary Fig. 15). The cell with the GO-g-PAM@PP separator still maintains an average Coulombic efficiency of $98 \%$ over 150 cycles under an increased current density of $1 \mathrm{~mA} \mathrm{~cm}^{-2}$, which is superior to those of the cells with PP and GO@PP separators and favorably comparable with those of previously reported asymmetric cells with Li metal anodes stabilized by various strategies (Fig. 4a, d and Supplementary Fig. 16) 17,36-38,42-48. To further demonstrate the unique merits of GO- $g$-PAM molecular brushes, a $\mathrm{Li} \mid \mathrm{Cu}$ cell with a separator coated with a mixture of GO and PAM, denoted as GO/PAM@PP, is also investigated under identical conditions. As shown in Supplementary Fig. 16, the GO-g-PAM@PP separator greatly outperforms the GO/PAM@PP separator for Li plating/ stripping. The results demonstrate that the molecular-level uniform distribution of polar functional groups enabled by the GO-g-PAM molecular brushes is crucial for achieving dendritefree deposition of Li metal, which results in stable SEI layers with minimized side reactions between the deposited $\mathrm{Li}$ and the electrolyte.

To further investigate the advantage of the GO-g-PAM@PP separator in the cycling stability of Li metal anodes, symmetric Li| $\mathrm{Li}$ cells with various separators are fabricated. As shown in Fig. 5a, when the cycling capacity is $1 \mathrm{mAh} \mathrm{cm}^{-2}$, the cell with the GO-g-PAM@PP separator delivers an excellent cycling stability with stable voltage plateaus for over $2600 \mathrm{~h}$ at a current
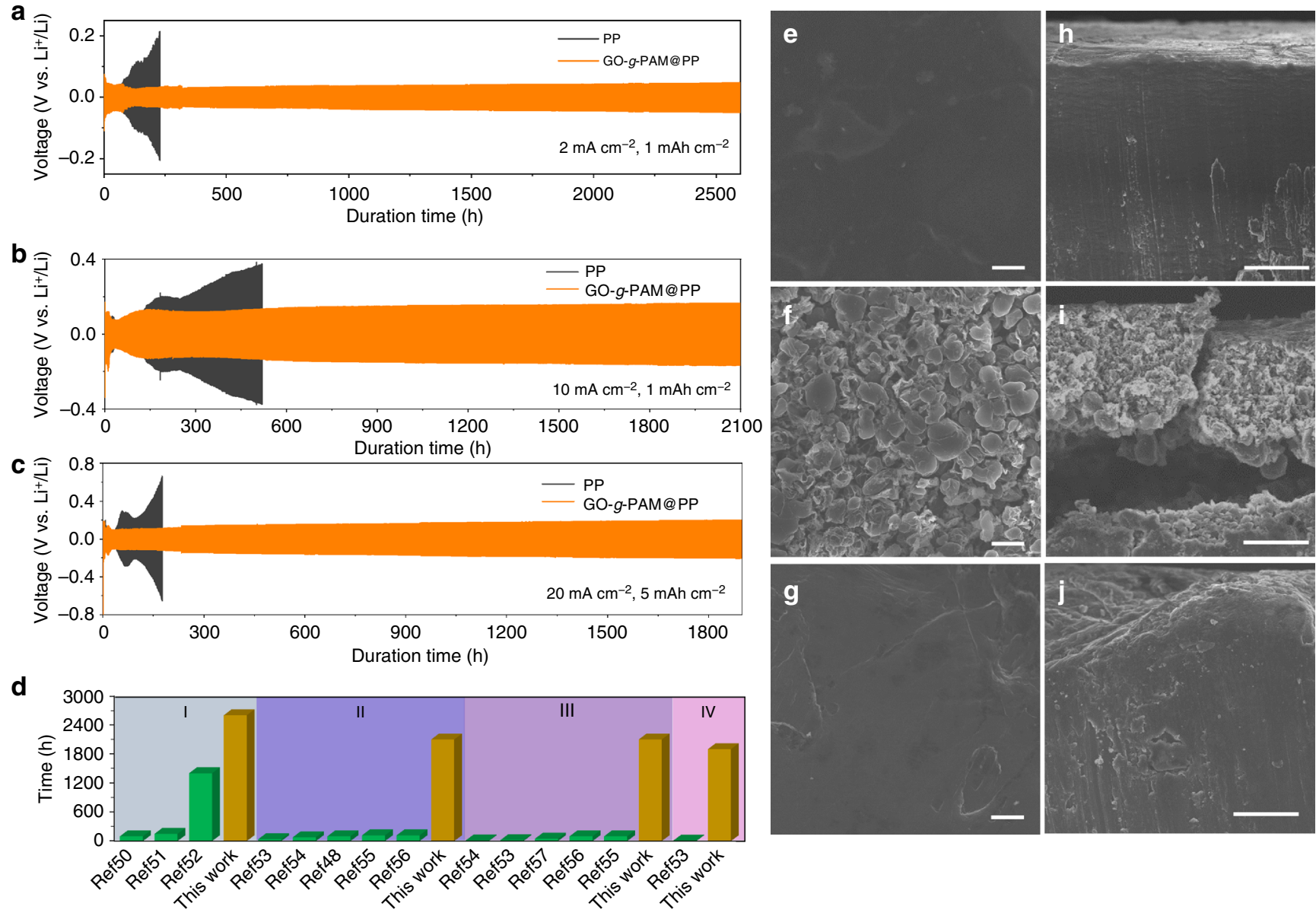

Fig. 5 Electrochemical performance of symmetric Li|Li cells with GO-g-PAM@PP and PP separators. Voltage-time profiles of Li plating/stripping processes with a cycling capacity of (a, b) 1 and (c) $5 \mathrm{mAh} \mathrm{cm}^{-2}$ at (a) 2, (b) 10, and (c) $20 \mathrm{~mA} \mathrm{~cm}^{-2}$ in symmetric Li|Li cells with GO-g-PAM@PP and PP separators. d Comparison of the cycle life of symmetric Li|Li cells with GO-g-PAM@PP separators and that of previously reported Li metal anodes stabilized by various strategies at the same current densities: (I) 2, (II) 5, (III) 10, and (IV) $20 \mathrm{~mA} \mathrm{~cm}^{-2}$. All symmetric cells were cycled at a cycling capacity of $1 \mathrm{mAh}$ cm ${ }^{-2}$, except that the cycling capacities were $0.4 \mathrm{mAh} \mathrm{cm}^{-2}$ for ref. ${ }^{51}$ and $2 \mathrm{mAh} \mathrm{cm}^{-2}$ for ref. 52 at $2 \mathrm{~mA} \mathrm{~cm}^{-2}$, and $5 \mathrm{mAh} \mathrm{cm}^{-2}$ for this work at $20 \mathrm{~mA} \mathrm{~cm}^{-2}$. e-g Top-view and $(\mathbf{h}-\mathbf{j})$ cross-section SEM images of $(\mathbf{e}, \mathbf{h})$ a fresh Li metal anode and Li metal anodes assembled with (f, i) PP and (g, j) GO-g-PAM@PP separators after 100 cycles. Scale bars: $(\mathbf{e}-\mathbf{g}) 10 \mu \mathrm{m}$ and $(\mathbf{h}-\mathbf{j}) 50 \mu \mathrm{m}$ 
density of $2 \mathrm{~mA} \mathrm{~cm}^{-2}$. In sharp contrast, the cell with the GO@PP separator exhibits a much larger voltage hysteresis (Supplementary Fig. 17); a gradual increase in voltage hysteresis is observed for the cell with the PP separator after cycling for only $73 \mathrm{~h}$ (Fig. 5a), which can be ascribed to the electrical disconnection and depletion of electrolyte resulting from the repeated growth/corrosion of $\mathrm{Li}$ dendrites and continuous consumption of electrolyte $^{49}$. Even at very high current densities of 5 and $10 \mathrm{~mA}$ $\mathrm{cm}^{-2}$, the cells with the GO-g-PAM@PP separators still exhibit long-term stability for over $2100 \mathrm{~h}$, greatly outperforming the cells with the PP separators (Fig. 5b and Supplementary Fig. 18). Remarkably, when the areal capacity is substantially increased to $5 \mathrm{mAh} \mathrm{cm}^{-2}$, which is much higher than that of current commercial batteries $\left(3 \mathrm{mAh} \mathrm{cm}^{-2}\right)$, stable $\mathrm{Li}$ plating/stripping of over $1900 \mathrm{~h}$ can still be achieved for the cell with the GO- $g$ PAM@PP separator at an extremely high current density of 20 $\mathrm{mA} \mathrm{cm}{ }^{-2}$, whereas the cell with the PP separator can only be stably cycled for no more than $36 \mathrm{~h}$ (Fig. 5c). The significant differences in cycle stability strongly demonstrate the superiority of GO-g-PAM@PP separator for repeated plating and stripping of Li. To the best of our knowledge, such an ultralong lifespan is superior to those of all previously reported $\mathrm{Li}$ anodes stabilized by various strategies under similar test conditions, which exhibit significantly decreased cycling performance when the current density is larger than $2 \mathrm{~mA} \mathrm{~cm}^{-2}$ (Fig. 5d) $48,50-57$. Moreover, the electrochemical performance of our GO- $g$-PAM@PP separators could be further optimized by tuning the structure of GO-g-PAM, e.g., the molecular weight of the PAM chains by changing the polymerization time (Supplementary Fig. 19).

It should be noted that the cells with GO-g-PAM@PP separators exhibit slight voltage fluctuations with a slightly larger voltage hysteresis than that of cells with PP separators in the initial cycles (Supplementary Fig. 20). This difference could be ascribed to SEI formation on the surfaces of GO-g-PAM molecular brushes that directly contact the Li foils (Supplementary Fig. 12a $)^{58,59}$. After the initial few cycles, the SEI is mature and stabilized, and thus the cells with GO-g-PAM@PP separators begin to demonstrate a flatter plateaus and smaller average voltage hysteresis in later long cycles. For further evidence, the voltage-time profile of a symmetric $\mathrm{Li} \mid \mathrm{Li} @ \mathrm{Cu}$ cell with a prestabilized GO-g-PAM@PP separator is also provided. As shown in Supplementary Fig. 21, this cell demonstrates much more stable voltage plateaus with reduced overpotentials at $5 \mathrm{~mA} \mathrm{~cm}^{-2}$.

The nanomorphologies of $\mathrm{Li}$ metal anodes after symmetric cell cycles are investigated to further clarify the effect of the GO-g-PAM molecular brushes on the suppression of $\mathrm{Li}$ dendrites. As shown in Fig. 5e, h, the fresh Li metal anode presents a dense structure with a smooth surface. However, for the cell with the PP separator, the Li metal anode displays obvious wire-shaped Li dendrites after the first cycle, and loosely stacked mossy Li with a highly porous structure is formed after 10 cycles; this latter morphology becomes more significant after 50 and 100 cycles (Fig. 5f, i, and Supplementary Fig. 22). In sharp contrast, after coating the PP separator with GO$g$-PAM molecular brushes, uniform and dense Li deposition is realized and no dendrite formation is observed on the anode surface after the first cycle (Supplementary Fig. 23a, b). Importantly, the Li metal anode still retains a relatively dense and compact structure with a dendrite-free flat surface even after 50 and 100 cycles, highlighting the advantages of GO- $g$-PAM molecular brushes for favorable dendrite-free Li plating/stripping behavior (Fig. 5g, j, and Supplementary Fig. 23c-f).

To demonstrate the potential of the GO-g-PAM@PP separator in practical $\mathrm{Li}$ metal batteries, the electrochemical performance of $\mathrm{Li} \mid \mathrm{Li}_{4} \mathrm{Ti}_{5} \mathrm{O}_{12}$ cells with GO-g-PAM@PP and PP separators is also investigated. As expected, the $\mathrm{Li} \mid \mathrm{Li}_{4} \mathrm{Ti}_{5} \mathrm{O}_{12}$ cell with the GO$g$-PAM@PP separator demonstrates better cycling performance than that with the PP separator. Specifically, the cell with the GO$g$-PAM@PP separator delivers a specific capacity of $160 \mathrm{mAh} \mathrm{g}^{-1}$ with a Coulombic efficiency of $96 \%$ at the first cycle and retains $77 \%$ of the initial capacity with a steady Coulombic efficiency of nearly $100 \%$ over 800 cycles (Fig. 6a, b). In comparison, the Coulombic efficiency of the $\mathrm{Li} \mid \mathrm{Li}_{4} \mathrm{Ti}_{5} \mathrm{O}_{12}$ cell with the $\mathrm{PP}$
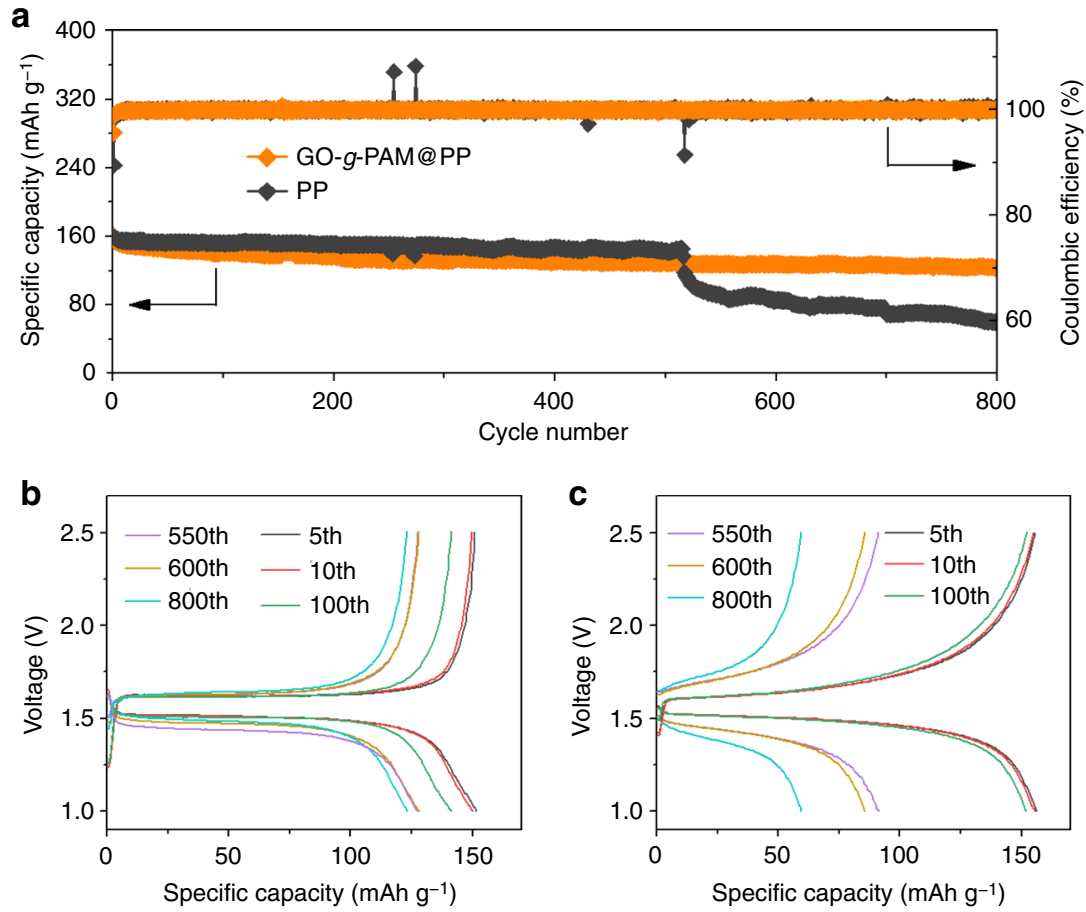

Fig. 6 Electrochemical performance of $\mathrm{Li}_{\mathrm{L}} \mathrm{Li}_{4} \mathrm{Ti}_{5} \mathrm{O}_{12}$ cells with GO-g-PAM@PP and PP separators. a Long-term cycling stability of Li|Li $\mathrm{L}_{4} \mathrm{Ti}_{5} \mathrm{O}_{12}$ cells with GOg-PAM@PP and PP separators at a current density of $3 \mathrm{C}\left(1 \mathrm{C}=175 \mathrm{~mA} \mathrm{~g}^{-1}\right)$. Galvanostatic charge-discharge profiles of $\mathrm{Li}_{\mid} \mathrm{Li}_{4} \mathrm{Ti}_{5} \mathrm{O}_{12}$ cells with $(\mathbf{b}) \mathrm{GO}-\mathrm{g}$ PAM@PP and (c) PP separators at 3C 
separator during the first cycle is only $90 \%$, and the efficiency exhibits occasional fluctuations during the cycles. In addition, the capacity of the $\mathrm{Li} \mid \mathrm{Li}_{4} \mathrm{Ti}_{5} \mathrm{O}_{12}$ cell with the PP separator suddenly degrades after 515 cycles, accompanied by a significantly increased overall overpotential (Fig. 6a, c). The results can be ascribed to the increasingly thicker SEI layer and "drying-out" of the cell caused by the continuous reaction of electrolyte with the uncontrollably deposited $\mathrm{Li}$ dendrites. The $\mathrm{Li} \mid \mathrm{Li}_{4} \mathrm{Ti}_{5} \mathrm{O}_{12}$ cell with the GO-g-PAM@PP separator exhibits a slightly larger capacity decay than that of the cell with the PP separator in the initial cycles, which could be ascribed to the irreversible formation of SEI films in the initial cycles on the surfaces of the GO-g-PAM molecular brushes that directly contact the $\mathrm{Li}$ foils (Supplementary Fig. 12a).

\section{Discussion}

Uniform and stable Li deposition is regulated by employing 2D molecular brush-functionalized porous bilayer composite separators, which are facilely obtained by coating the GO-g-PAM molecular brushes on commercial PP separators with an upscalable blade-coating procedure. Benefiting from the abundant polar functional groups of hairy PAM chains, the interconnected interspaces between the stacked 2D molecular brushes, and the robust mechanical strength of GO nanosheets, the functional porous bilayer composite separators not only possess excellent mechanical strength but also facilitate molecular-level homogeneous and fast $\mathrm{Li}$ ionic flux on the surfaces of electrodes. These unique features of the functional porous bilayer composite separators enable dendrite-free uniform Li deposition with high Coulombic efficiency at Li metal anodes as well as ultralong-term reversible Li plating/stripping at ultrahigh current densities.

\section{Methods}

Synthesis of GO-Br. In a typical synthesis process, $100 \mathrm{mg}$ of GO (purchased from Hangzhou Gaoxi Technology Co., Ltd., China) dispersed in $190 \mathrm{~mL}$ of N,Ndimethylformamide (DMF) was added to a three-necked round-bottom flask $(250 \mathrm{~mL})$ and sonicated for $0.5 \mathrm{~h}$, followed by adding $1.3 \mathrm{~mL}$ of triethanolamine. After cooling to $0^{\circ} \mathrm{C}$ and purging with $\mathrm{N}_{2}$ for $30 \mathrm{~min}$, a mixed solution of $\alpha$ bromoisobutyryl bromide $(0.857 \mathrm{~mL})$ and DMF $(10 \mathrm{~mL})$ was added dropwise for $0.5 \mathrm{~h}$. The reaction was carried out under stirring at $0^{\circ} \mathrm{C}$ for $2 \mathrm{~h}$, followed by $30^{\circ} \mathrm{C}$ for $24 \mathrm{~h}$. The resulting GO-Br was then centrifuged at 12,000 rpm, washed with deionized water three times, and redispersed in $100 \mathrm{~mL}$ of deionized water.

Synthesis of GO-g-PAM molecular brushes. Typically, 2,2'-bipyridine ( $0.0413 \mathrm{~g}$ ) and acrylamide $(0.9467 \mathrm{~g})$ were first added to the above $\mathrm{GO}-\mathrm{Br}$ aqueous dispersion and stirred in a Schlenk flask under a $\mathrm{N}_{2}$ atmosphere for $30 \mathrm{~min}$. A total of $0.0187 \mathrm{~g}$ of $\mathrm{CuBr}$ was then added to the mixture, and the solution was continually stirred under a $\mathrm{N}_{2}$ atmosphere for another $10 \mathrm{~min}$. Afterwards, the reaction was carried out at $70{ }^{\circ} \mathrm{C}$ for $12 \mathrm{~h}$. The polymerization was stopped by opening the flask and exposing the catalyst to air. The resulting GO-g-PAM was centrifuged at 12,000 rpm for $10 \mathrm{~min}$, washed with deionized water three times, and then freeze-dried for $24 \mathrm{~h}$. GO-g-PAM molecular brushes with different molecular weights of PAM chains were also prepared by controlling the polymerization times.

Synthesis of PAM. A total of $7.815 \mathrm{~g}$ of acrylamide, $0.1563 \mathrm{~g}$ of $2,2^{\prime}$-azobis-(2methylpropionitrile), $45 \mathrm{~mL}$ of ethanol, and $45 \mathrm{~mL}$ of acetone were added into a three-necked round-bottom flask $(150 \mathrm{~mL})$ under a $\mathrm{N}_{2}$ atmosphere with stirring for $30 \mathrm{~min}$. The flask was then immersed in a water bath at $60^{\circ} \mathrm{C}$ for $3 \mathrm{~h}$. Afterwards, the precipitates were filtered and washed with ethanol three times, followed by vacuum drying at $40{ }^{\circ} \mathrm{C}$ overnight.

Fabrication of functional porous bilayer composite separators. Typically, GO$g$-PAM and polyvinylidene difluoride (PVDF) binder with a mass ratio of 80:20 were mixed in N-methyl-2-pyrrolidinone (NMP) and then coated onto one side of a commercial PP separator (Celgard 2325) with a doctor blade, followed by vacuum drying at $50{ }^{\circ} \mathrm{C}$ overnight. After that, the GO- $g$-PAM@PP separators were punched into disks with a diameter of $19 \mathrm{~mm}$. Control composite separators, including GO@PP, PAM@PP, and GO/PAM@PP, were prepared by replacing GO-g-PAM with GO, PAM, and the mixture of GO and PAM with the same PAM content as GO-g-PAM, respectively, under identical fabrication conditions.
Material characterization. The nanomorphologies were visualized by fieldemission scanning electron microscopy (FESEM, S-4800). Elemental mapping was achieved by transmission electron microscopy (TEM, Tecnai G2 Spirit). A Micromeritics ASAP 2020 surface area and porosity analyzer was used to analyze the pore structure of the separators, and the PSD was obtained by using a DFT model. FTIR spectra were recorded at room temperature on a Bruker Equinox 55 FTIR spectroscope. Contact angle measurements were conducted by using a KRUSS DSA100 machine. The Young's moduli of the separators were measured by AFM (Bruker Dimension Fastscan Bio) in peak force quantitative nanomechanics mode and analyzed by the Derjaguin-Muller-Toporov model.

Electrochemical measurements. All electrochemical measurements were conducted by using CR2032 coin cells at room temperature. The electrolyte used for $\mathrm{Li}$ deposition was $30 \mu \mathrm{L}$ of $1.0 \mathrm{M}$ LiTFSI in a mixture of 1,3-dioxalane and dimethyl ether (1:1 by volume) with $1 \mathrm{wt} \% \mathrm{LiNO}_{3}$ additive, while the electrolyte used for $\mathrm{Li}$ $\mathrm{Li}_{4} \mathrm{Ti}_{5} \mathrm{O}_{12}$ cells was $1.0 \mathrm{M} \mathrm{LiPF}_{6}$ in a 50:50 (w/w) mixture of ethylene carbonate and diethyl carbonate. LSV measurements were collected with Li foil as the counter electrode, a stainless-steel disc as the working electrode, and the tested separator sandwiched between the electrodes with the coated side toward the Li foil. Electrochemical impedance spectra were measured with symmetric $\mathrm{Li} \mid \mathrm{Li}$ cells by applying an AC amplitude of $5 \mathrm{mV}$ over a frequency range from 0.01 to $10^{5} \mathrm{~Hz}$. The asymmetric $\mathrm{Li} \mid \mathrm{Cu}$ cells were assembled with the coated side of the composite separators toward the $\mathrm{Cu}$ foils, and then cycled from 0 to $1 \mathrm{~V}$ at $50 \mu \mathrm{A}$ for five cycles to stabilize the interface. The Coulombic efficiency was calculated as the ratio of the stripping versus the plating capacity. The symmetric $\mathrm{Li} \mid \mathrm{Li}$ cells were assembled with two Li foils as the working electrode and counter electrode, respectively, and PP or GO-g-PAM@PP separators as the separators. To prepare the $\mathrm{Li}_{4} \mathrm{Ti}_{5} \mathrm{O}_{12}$ electrode, $\mathrm{Li}_{4} \mathrm{Ti}_{5} \mathrm{O}_{12}$, acetylene black, and PVDF at a weight ratio of 7:2:1 were mixed in NMP to form a homogeneous slurry, and then rigorously pasted on a $\mathrm{Cu}$ foil. After drying at $60^{\circ} \mathrm{C}$ under vacuum for $12 \mathrm{~h}$, the $\mathrm{Li} \mid \mathrm{Li}_{4} \mathrm{Ti}_{5} \mathrm{O}_{12}$ cells were assembled with PP or GO-g-PAM@PP separators. The cells were galvanostatically cycled between 1.0 and $2.5 \mathrm{~V}$ at $3 \mathrm{C}\left(1 \mathrm{C}=175 \mathrm{~mA} \mathrm{~g}^{-1}\right)$.

\section{Data availability}

The authors declare that all the data supporting the findings of this study are available within the article and its Supplementary Information or from the corresponding author upon reasonable request.

Received: 23 December 2018 Accepted: 25 February 2019 Published online: 25 March 2019

\section{References}

1. Han, J. et al. Caging tin oxide in three-dimensional graphene networks for superior volumetric lithium storage. Nat. Commun. 9, 402 (2018).

2. Zhang, Q., Li, F., Huang, J. Q. \& Li, H. Lithium-sulfur batteries: co-existence of challenges and opportunities. Adv. Funct. Mater. 28, 1804589 (2018).

3. Wang, A. et al. Horizontal centripetal plating in the patterned voids of Li/ graphene composites for stable lithium-metal anodes. Chem 4, 2192-2200 (2018).

4. Wu, Z. S. et al. Bottom-up fabrication of sulfur-doped graphene films derived from sulfur-annulated nanographene for ultrahigh volumetric capacitance micro-supercapacitors. J. Am. Chem. Soc. 139, 4506-4512 (2017).

5. Fang, R. et al. More reliable lithium-sulfur batteries: status, solutions and prospects. Adv. Mater. 29, 1606823 (2017).

6. Sun, Z. et al. Conductive porous vanadium nitride/graphene composite as chemical anchor of polysulfides for lithium-sulfur batteries. Nat. Commun. 8, 14627 (2017).

7. Wu, X., Wang, Z., Yu, M., Xiu, L. \& Qiu, J. Stabilizing the MXenes by carbon nanoplating for developing hierarchical nanohybrids with efficient lithium storage and hydrogen evolution capability. Adv. Mater. 29, 1607017 (2017).

8. Kaskhedikar, N. A. \& Maier, J. Lithium storage in carbon nanostructures. $A d v$. Mater. 21, 2664-2680 (2009).

9. Ellis, B. L., Lee, K. T. \& Nazar, L. F. Positive electrode materials for Li-ion and Li-batteries. Chem. Mater. 22, 691-714 (2010).

10. Bruce, P. G., Freunberger, S. A., Hardwick, L. J. \& Tarascon, J. M. Li- $\mathrm{O}_{2}$ and Li-S batteries with high energy storage. Nat. Mater. 11, 19-29 (2012).

11. Lin, D., Liu, Y. \& Cui, Y. Reviving the lithium metal anode for high-energy batteries. Nat. Nanotechnol. 12, 194-206 (2017).

12. Guo, Y., Li, H. \& Zhai, T. Reviving lithium-metal anodes for next-generation high-energy batteries. Adv. Mater. 29, 1700007 (2017).

13. Liu, Y. et al. Making Li-metal electrodes rechargeable by controlling the dendrite growth direction. Nat. Energy 2, 17083 (2017).

14. Tu, Z. et al. Fast ion transport at solid-solid interfaces in hybrid battery anodes. Nat. Energy 3, 310-316 (2018).

15. Yan, C. et al. Dual-layered film protected lithium metal anode to enable dendrite-free lithium deposition. Adv. Mater. 30, 1707629 (2018). 
16. Ding, F. et al. Dendrite-free lithium deposition via self-healing electrostatic shield mechanism. J. Am. Chem. Soc. 135, 4450-4456 (2013).

17. Zhang, R. et al. Lithiophilic sites in doped graphene guide uniform lithium nucleation for dendrite-free lithium metal anodes. Angew. Chem. Int. Ed. 56, 7764-7768 (2017).

18. Yun, Q. et al. Chemical dealloying derived 3D porous current collector for $\mathrm{Li}$ metal anodes. Adv. Mater. 28, 6932-6939 (2016).

19. Suo, L., Hu, Y. S., Li, H., Armand, M. \& Chen, L. A new class of Solvent-inSalt electrolyte for high-energy rechargeable metallic lithium batteries. Nat. Commun. 4, 1481 (2013).

20. Stark, J. K., Ding, Y. \& Kohl, P. A. Dendrite-free electrodeposition and reoxidation of lithium-sodium alloy for metal-anode battery. J. Electrochem. Soc. 158, A1100-A1105 (2011).

21. Liang, Y. et al. Fabrication and nanostructure control of super-hierarchical carbon materials from heterogeneous bottlebrushes. Chem. Sci. 8, 2101-2106 (2017).

22. Lu, Y., Tu, Z. \& Archer, L. A. Stable lithium electrodeposition in liquid and nanoporous solid electrolytes. Nat. Mater. 13, 961-969 (2014).

23. Zhao, Q. et al. Building organic/inorganic hybrid interphases for fast interfacial transport in rechargeable metal batteries. Angew. Chem. Int. Ed. 57, 992-996 (2018).

24. Liu, S. et al. Crumpled graphene balls stabilized dendrite-free lithium metal anodes. Joule 2, 184-193 (2018).

25. Liu, L. et al. Free-standing hollow carbon fibers as high-capacity containers for stable lithium metal anodes. Joule 1, 563-575 (2017).

26. Gao, Y. et al. Interfacial chemistry regulation via a skin-grafting strategy enables high-performance lithium-metal batteries. J. Am. Chem. Soc. 139, 15288-15291 (2017).

27. Kozen, A. C. et al. Next-generation lithium metal anode engineering via atomic layer deposition. ACS Nano 9, 5884-5892 (2015).

28. Xu, R. et al. Artificial soft-rigid protective layer for dendrite-free lithium metal anode. Adv. Funct. Mater. 28, 1705838 (2018).

29. Zhao, C. Z. et al. An ion redistributor for dendrite-free lithium metal anodes. Sci. Adv. 4, eaat3446 (2018).

30. Wu, H., Cao, Y., Su, H. \& Wang, C. Tough gel electrolyte using double polymer network design for the safe, stable cycling of lithium metal anode. Angew. Chem. Int. Ed. 57, 1361-1365 (2018).

31. Lin, D. et al. A silica-aerogel-reinforced composite polymer electrolyte with high ionic conductivity and high modulus. Adv. Mater. 30, 1802661 (2018)

32. Zhao, C. Z. et al. An anion-immobilized composite electrolyte for dendritefree lithium metal anodes. Proc. Natl Acad. Sci. USA 114, 11069 (2017).

33. Lu, Q. et al. Dendrite-free, high-rate, long-life lithium metal batteries with a 3D cross-linked network polymer electrolyte. Adv. Mater. 29, 1604460 (2017).

34. Yan, K. et al. Ultrathin two-dimensional atomic crystals as stable interfacia layer for improvement of lithium metal anode. Nano. Lett. 14, 6016-6022 (2014).

35. Kim, J. S., Kim, D. W., Jung, H. T. \& Choi, J. W. Controlled lithium dendrite growth by a synergistic effect of multilayered graphene coating and an electrolyte additive. Chem. Mater. 27, 2780-2787 (2015).

36. Cheng, X. B. et al. Dendrite-free lithium deposition induced by uniformly distributed lithium ions for efficient lithium metal batteries. Adv. Mater. 28, 2888-2895 (2016).

37. Liang, Z. et al. Polymer nanofiber-guided uniform lithium deposition for battery electrodes. Nano. Lett. 15, 2910-2916 (2015).

38. Liu, W. et al. Functional metal-organic framework boosting lithium metal anode performance via chemical interactions. Chem. Sci. 8, 4285-4291 (2017).

39. Foroozan, T. et al. Synergistic effect of graphene oxide for impeding the dendritic plating of Li. Adv. Funct. Mater. 28, 1705917 (2018).

40. Li, G. et al. Stable metal battery anodes enabled by polyethylenimine sponge hosts by way of electrokinetic effects. Nat. Energy 3, 1076-1083 (2018).

41. Yang, C. P., Yin, Y. X., Zhang, S. F., Li, N. W. \& Guo, Y. G. Accommodating lithium into $3 \mathrm{D}$ current collectors with a submicron skeleton towards long-life lithium metal anodes. Nat. Commun. 6, 8058 (2015)

42. Xue, P. et al. A hierarchical silver-nanowire-graphene host enabling ultrahigh rates and superior long-term cycling of lithium-metal composite anodes. Adv. Mater. 30, 1804165 (2018).

43. Zuo, T. T. et al. Graphitized carbon fibers as multifunctional 3D current collectors for high areal capacity Li anodes. Adv. Mater. 29, 1700389 (2017).

44. Liu, Y. et al. An artificial solid electrolyte interphase with high Li-ion conductivity, mechanical strength, and flexibility for stable lithium metal anodes. Adv. Mater. 29, 1605531 (2017).

45. Liu, K. et al. Lithium metal anodes with an adaptive "solid-liquid" interfacial protective layer. J. Am. Chem. Soc. 139, 4815-4820 (2017).

46. Zheng, G. et al. Interconnected hollow carbon nanospheres for stable lithium metal anodes. Nat. Nanotechnol. 9, 618-623 (2014).

47. Pang, Q., Zhou, L. \& Nazar, L. F. Elastic and Li-ion-percolating hybrid membrane stabilizes Li metal plating. Proc. Natl Acad. Sci. USA 115, 12389-12394 (2018).
48. Zhang, W., Zhuang, H. L., Fan, L., Gao, L. \& Lu, Y. A. “cation-anion regulation" synergistic anode host for dendrite-free lithium metal batteries. Sci. Adv. 4, eaar4410 (2018)

49. Cheng, X. B. et al. Dual-phase lithium metal anode containing a polysulfideinduced solid electrolyte interphase and nanostructured graphene framework for lithium-sulfur batteries. ACS Nano 9, 6373-6382 (2015).

50. Ye, H. et al. Stable Li plating/stripping electrochemistry realized by a hybrid $\mathrm{Li}$ reservoir in spherical carbon granules with $3 \mathrm{D}$ conducting skeletons. J. Am. Chem. Soc. 139, 5916-5922 (2017).

51. Cheng, X. B. et al. Nanodiamonds suppress the growth of lithium dendrites. Nat. Commun. 8, 336 (2017).

52. Liang, X. et al. A facile surface chemistry route to a stabilized lithium metal anode. Nat. Energy 2, 17119 (2017).

53. Wang, H., Lin, D., Liu, Y., Li, Y. \& Cui, Y. Ultrahigh-current density anodes with interconnected $\mathrm{Li}$ metal reservoir through overlithiation of mesoporous $\mathrm{AlF}_{3}$ framework. Sci. Adv. 3, e1701301 (2017)

54. Deng, W., Zhou, X., Fang, Q. \& Liu, Z. Microscale lithium metal stored inside cellular graphene scaffold toward advanced metallic lithium anodes. Adv. Energy Mater. 8, 1703152 (2018).

55. Zhang, H. et al. Lithiophilic-lithiophobic gradient interfacial layer for a highly stable lithium metal anode. Nat. Commun. 9, 3729 (2018).

56. Zhao, J. et al. Surface fluorination of reactive battery anode materials for enhanced stability. J. Am. Chem. Soc. 139, 11550-11558 (2017).

57. Fan, L. et al. Stable lithium electrodeposition at ultra-high current densities enabled by 3D PMF/Li composite anode. Adv. Energy Mater. 8, 1703360 (2018).

58. Zhang, R. et al. Coralloid carbon fiber-based composite lithium anode for robust lithium metal batteries. Joule 2, 764-777 (2018).

59. Xie, J. et al. Incorporating flexibility into stiffness: self-grown carbon nanotubes in melamine sponges enable a lithium-metal-anode capacity of $15 \mathrm{~mA} \mathrm{~h} \mathrm{~cm}^{-2}$ cyclable at $15 \mathrm{~mA} \mathrm{~cm}^{-2}$. Adv. Mater. 31, 1805654 (2019).

\section{Acknowledgements}

This work was supported by the National Natural Science Foundation of China (U1601206, 51702370, 51872336, and 51672313), Leading Scientific, Technical and Innovation Talents of Guangdong Special Support Program (2017TX04C248), Fundamental Research Funds for the Central Universities (18lgzd10, 17lgpy83), and National Program for Support of Top-notch Young Professionals

\section{Author contributions}

D.W. conceived the concept and directed the research. S.L and D.W. designed the project. C.L., C.S., and G.L. carried out material synthesis. C.L., S.L., C.S., G.L., and Z.L. performed material characterization and electrochemical tests. C.L., S.L., and D.W. wrote the paper. R.F. gave advice on the research. All authors discussed the results and commented on the paper.

\section{Additional information}

Supplementary Information accompanies this paper at https://doi.org/10.1038/s41467 019-09211-z.

Competing interests: The authors declare no competing interests.

Reprints and permission information is available online at http://npg.nature.com/ reprintsandpermissions/

Journal peer review information: Nature Communications thanks Liqiang Mai and the other anonymous reviewer(s) for their contribution to the peer review of this work.

Publisher's note: Springer Nature remains neutral with regard to jurisdictional claims in published maps and institutional affiliations.

Open Access This article is licensed under a Creative Commons Attribution 4.0 International License, which permits use, sharing, adaptation, distribution and reproduction in any medium or format, as long as you give appropriate credit to the original author(s) and the source, provide a link to the Creative Commons license, and indicate if changes were made. The images or other third party material in this article are included in the article's Creative Commons license, unless indicated otherwise in a credit line to the material. If material is not included in the article's Creative Commons license and your intended use is not permitted by statutory regulation or exceeds the permitted use, you will need to obtain permission directly from the copyright holder. To view a copy of this license, visit http://creativecommons.org/ licenses/by/4.0/.

(c) The Author(s) 2019 